\section{A simple method for recording thin-layer acrylamide gel electropherograms}

U HLA-PE AND U TIN-WIN Biochemistry Research Division, Department of Medical Research, No. 5, Zafar Shah Road, Dagon PO, Rangoon, Burma

Thin-layer acrylamide gel electrophoretic technique, based on the method described by Nandi and Lewis, ${ }^{1}$ has been used in our laboratory for the electrophoresis of blood and malaria parasite enzymes. It was found that preservation of the enzyme electropherograms in acid solution is cumbersome, space-occupying, and unstable. Alternative methods for permanent recording of the electropherograms are direct scanning of the gels in a densitometer and photographic techniques. These techniques require extra equipment and skill. In this paper we describe a simple procedure for recording electropherograms of enzyme patterns in their original size and colour.

\section{Method}

Electrophoresis is carried out by using parasite enzyme extracts or haemolysates on $5.5 \mathrm{~g} \%$ acrylamide gel plates $(2 \times 120 \times 165 \mathrm{~mm})$. After electrophoresis, the gel is carefully detached from the glass plate under ice-cold water using a blunt, thin spatula. The gel is stained with an appropriate MTT-linked enzyme substrate staining solution. ${ }^{2}$ The stained gel is washed with phosphate-buffered saline ( $\mathrm{pH} 7 \cdot 4 ; 0 \cdot 15 \mathrm{M})$ to stop the enzymic action and staining. The washed gel slab is then placed on a Perspex sheet or paper-thin plastic sheet which has been placed on the base of a metallic tray. Excess buffer is gently drained off by tilting the base plate. The gel is covered with a strip of Whatman filter paper No. 1 , which is slightly larger than the size of the gel. The filter paper is well flattened out on the surface of the gel by the middle finger, removing air bubbles at the same time. The gel sandwich thus prepared is dried at room temperature or at $37^{\circ} \mathrm{C}$

Received for publication 12 February 1980

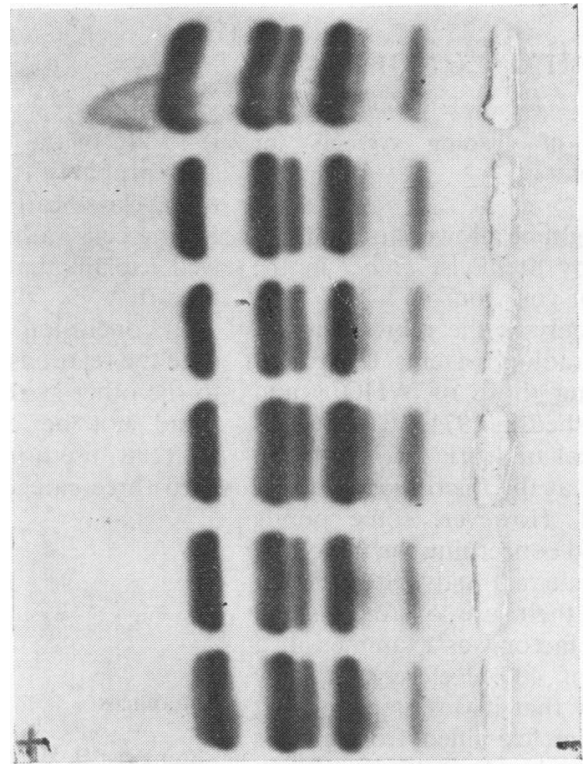

Recorded electropherogram of human red blood cell lactic dehydrogenase pattern. Staining: Reduction of MTT tetrazolium.

overnight. The dried gel is well adhered to the filter paper, forming a glossy-type of coloured electropherogram which has been filed and stored more than six months without deterioration. The electropherogram retains the original size and colour of the enzyme patterns (Figure).

We express our thanks to Ko Sein Min for his able technical assistance.

\section{References}

${ }^{1}$ Nandi M, Lewis GP. Thin-layer acrylamide gel electrophoresis. J Clin Pathol 1970;23:727-9.

2 Carter R, McGregor IA. Enzyme variation in Plasmodium falciparum in the Gambia. Trans Royal Soc Trop Med Hyg 1973;67:830-7.

Requests for reprints to: Dr U Hla-Pe, Head, Biochemistry Research Division, Department of Medical Research, No. 5 Zafar Shah Road, Rangoon, Burma. 